\title{
Estudio preliminar sobre la desintoxicación ambulatoria de 18 pacientes alcohólicos con Tratamiento de Observación Directa
}

\author{
Ramón lloréns Martínez*; María Calatayud Francés**; Esperanza Morales Gallús***; \\ Consol Añó Cervera***; Librada Adriá Caballero****. \\ * Médico-Coordinador de la Unidad de Conductas Adictivas del Área 10 de Salud de Alzira (Valencia). \\ * Psicóloga de la Unidad de Conductas Adictivas del Área 10 de Salud de Alzira (Valencia). \\ *** Médico de la Unidad de Conductas Adictivas del Área 9 de Salud de Catarroja (Valencia). \\ ****Enfermera de la Unidad de Conductas Adictivas del Área 10 de Salud de Alzira (Valencia). \\ *****Auxiliar de Enfermería de la Unidad de Conductas Adictivas del Área 10 de Salud de Alzira (Valencia). \\ Enviar correspondencia a: \\ Ramón Lloréns Martínez, Unidad de Conductas Adictivas, Centro de Salud de San Isidro.
}

Avda. Campos Crespo / José Andreu Alabarta s/n, 46014-Valencia (España) ralloma@comv.es

\section{RESUMEN}

Los Tratamientos de Observación Directa (TOD) han sido probados en diferentes patologías. No tenemos constancia de que en la desintoxicación ambulatoria de alcohol se hayan utilizado. El objetivo de este trabajo es comprobar si una Unidad de Conductas Adictivas (UCA) puede, ambulatoriamente, desintoxicar y disminuir el riesgo de recaída precoz (doce semanas) en pacientes alcohólicos, con una dependencia orgánica establecida, con un síndrome de abstinencia grave, con múltiples tratamientos previos y que no habían conseguido mantener la abstinencia durante un periodo continuado de tres meses en los últimos dos años, y hacerlo mediante un modelo de abordaje multimodal médico-psicológico y de enfermería basado en una visita diaria breve y supervisión farmacológica.

Los resultados obtenidos fueron los siguientes: de los dieciocho pacientes incluidos en este estudio, a las doce semanas, trece (72\%) permanecían abstinentes, cuatro (22\%) recayeron, uno abandonó y diecisiete (94\%) se mantenían adheridos a tratamiento. Se analizan las características de los pacientes abstinentes, de los que recayeron (4) y del abandono (1). El CIWA-Ar medio se situó en 27.05 (21-36), por encima de 20 se considera síndrome de abstinencia grave, a pesar de lo cual no se produjo ningún acontecimiento adverso que requiriera ingreso hospitalario. La adherencia conseguida (94 \%) favoreció que, los pacientes más deteriorados y con menores recursos, pudieren beneficiarse no solo de algunos programas sociales sino también de los servicios básicos de salud con la consiguiente mejoría en su calidad de vida.

Palabras clave: Tratamiento de observación directa, desintoxicación ambulatoria, recaída precoz (12 semanas), adherencia.

\section{ABSTRACT}

Directly Observed Treatment (TOD-DOT) has been tested in different conditions. The objective of this work is to check whether a UCA-CAB (Centre for Addictive Behaviour) can achieve detox and reduce the risk of early relapse (up to 12 weeks) in alcoholic patients. All patients had an established organic addiction and serious withdrawal syndrome, and had undergone multiple previous treatments. Furthermore, they had not managed to abstain for a 3-month consecutive period over the previous 2 years. The aim of the Directly Observed Treatment was to attain detox and reduce relapse by following a multi-method approach: medical, psychological and personal care, based on a brief daily consultation and pharmacological supervision.

The results were as follows: Of the 18 patients included in the study, after 12 weeks, 13 (72\%) were still abstinent and 4 (22 \%) had relapsed. Thus, 17 (94\%) were still following the treatment, with just one drop-out. We analysed the profiles of the patients abstaining, of those who relapsed (4) and of the one who dropped out. The average CIWA-Ar was 27.05 (21-36). Any value over 20 is considered to indicate serious withdrawal syndrome, though there were no negative events leading to hospitalization. Level of adherence to the treatment (94\%) meant that the most seriously affected patients and those with fewest financial resources could benefit, not only from any auxiliary social schemes, but also from basic health services, permitting them to improve the quality of their everyday life.

Key words: Directly Observed Treatment, Detox (via CABoutpatient care units), early relapse (up to 12 weeks), adherence. 


\section{INTRODUCCIÓN}

$\mathbf{L}$ a recaída es el sine qua non del alcoholismo'. En el caso del alcoholismo su tratamiento se caracteriza por la presencia de recaídas, por ser largo $y$, sobre todo, por la existencia de importantes tasas de abandono ${ }^{2}$. Con relación a la historia natural de la dependencia alcohólica en nuestro entorno..., alrededor de un tercio de los que inician tratamiento recaen durante los tres primeros meses ${ }^{3}$. La dependencia al alcohol es una enfermedad progresiva, crónica y altamente recidivante que por su elevada prevalencia y su alta morbi-mortalidad se ha convertido en uno de los principales problemas de salud pública en nuestro medio ${ }^{4}$. En este sentido un trabajo publicado recientemente sobre el impacto del alcohol en España y Euro$\mathrm{pa}^{5}$, viene a poner de manifiesto la responsabilidad que tiene el alcohol en las muertes prematuras y en la discapacidad (12\%) entre los hombres y el (2\%) entre las mujeres, la elevada tasa de consumidores de riesgo de alcohol (15\% de la población de Europa), y la prevalencia de dependencia de alcohol o alcoholismo que alcanza al $5 \%$ de europeos y al $1 \%$ de europeas. Ante todo ello, las medidas preventivas que, hasta el momento, se han mostrado más eficaces han sido las que limitan la disponibilidad de alcohol (aumento del precio de las bebidas alcohólicas, reducción de horarios y lugares de venta, disminución de la tasa de alcoholemia permitida para la conducción de vehículos, la exigencia de la mayoría de edad para la venta y consumo de alcohol y la reducción de su publicidad y patrocinio). Posiblemente, la dificultad en la penetración de los mensajes preventivos en el marco educativo, deba hacernos reflexionar sobre la capacidad de impacto y verosimilitud que tiene para nuestros jóvenes un mensaje saludable, en el contexto de un comportamiento sociológico que, en muchas ocasiones y a cualquier precio, rinde muchos valores éticos y de convivencia a la competitividad y ganancia desbocadas.

La CIE-106 enuncia en F10: Trastornos mentales y del comportamiento debidos al consumo de alcohol; $y$ en F1x.2 describe el: Síndrome de dependencia y las pautas para su diagnóstico. Como la CIE-10 establece, el síndrome de abstinencia en general y del alcohol en particular, es "un conjunto de manifestaciones fisiológicas, comportamentales y cognoscitivas en el cual el consumo, para el caso de una sustancia, adquiere la máxima prioridad para el individuo", el deseo de consumo es "fuerte y a veces insuperable" $y$, cuando la recaída irrumpe, "después de un periodo de abstinencia lleva a la instauración más rápida del resto de características del síndrome de lo que sucede en individuos no dependientes".

Entre los instrumentos de evaluación de la dependencia alcohólica el MALT (Münchner Alkoholismus Test, diseñado por Feuerlein et al. ${ }^{7}$ y validado en España en 1984 por Rodríguez-Martos y Suárez ${ }^{8}$, por su elevada sensibilidad (100\%) y especificidad $(82 \%)$, puede utilizarse como cuestionario diagnóstico de alcoholismo 9 . Para nuestro estudio utilizamos, como instrumento diagnóstico el MALT. Este es un cuestionario que tiene la propiedad de captar problemas de alcoholismo en pacientes con alto grado de negación y se ha propuesto como una herramienta en pacientes con consumo de riesgo y perjudicial cuando se obtiene, previamente, una puntuación positiva en otros cuestionarios más breves como el CAGE, propuesto por Ewing y Rouse ${ }^{10}$ en 1970, y validado por Mayfield et al. ${ }^{11}$ en 1974, su versión española fue validada por Rodríguez-Martos et al. ${ }^{12}$ en 1986, o el CBA, ideado por Feuerlein ${ }^{13}$ en 1976 cuya versión española fue validada por Rodríguez-Martos et al..$^{12}$ en 1986. El MALT consta de dos partes: el MALT-S (MALT-Subjetivo) que estima la percepción subjetiva del paciente (dependencia psicológica) en relación a sus problemas con el alcohol, y el MALT-O (MALT-Objetivo) que informa sobre indicadores orgánicos de dependencia alcohólica (enfermedad hepática, polineuropatía, delirium tremens, consumo alcohólico superior a $150 \mathrm{ml}$, 120 en las mujeres, de alcohol puro al día al menos durante unos meses, consumo de alcohol superior a los $300 \mathrm{ml}$, en la mujer $240 \mathrm{ml}$, de alcohol puro una o más veces al mes, aliento enólico en el momento de la exploración médica y búsqueda anterior de ayuda por parte de familiares o allegados).

La medición cuantitativa del síndrome de abstinencia depende de la idoneidad de las escalas utilizadas para establecer el diagnóstico, estimar la necesidad de tratamiento farmacológico y determinar el momento adecuado para la reducción y la retirada de éste ${ }^{14}$. El síndrome de abstinencia alcohólica puede objetivarse mediante diversas escalas que permiten valorar la intensidad de la abstinencia, pero sin lugar a duda la CIWA (Clinical Institute Withdrawal Assessment for Alcohol Scale) y sus derivaciones (la CIWA-Ar, sobre todo $)^{15}$ son las más utilizadas para la valoración de la abstinencia, y se han usado tanto en el manejo clínico como en investigación. La CIWA-Ar, versión revisada de la CIWA, constituye el "patrón de oro" por ser sencilla y rápida a la hora de ser aplicada y su uso está muy extendido ${ }^{16}$.

Uno de los problemas más importantes a que han de hacer frente tanto los pacientes y su red social como los clínicos es el de la recaída. Muchos pacientes y sus familias se desesperan ante las continuas recaídas y pueden llegar a creer que no existe una salida satisfactoria. Los procesos de recaída son motivo de perplejidad para el propio adicto, porque la mayoría de veces se trata de un fenómeno que le sobrepasa y que escapa a su propio control, también son motivo de zozobra para su entorno y causa de frustración para los profesionales que trabajan en el ámbito de la clínica de conductas adictivas ${ }^{17}$. 
Los clínicos disponemos en la actualidad de tratamientos efectivos tanto médicos como psicológicos para el abordaje del alcoholismo. Es innegable, además, el interés por ampliar y encontrar nuevos fármacos capaces de actuar sobre aspectos clave de la patología adictiva como son la motivación, el craving, la impulsividad, la reducción del consumo y la prevención de recaídas, e incluir esta actuación farmacológica en el marco de un programa terapéutico multicomponente bio-psico-social del paciente adicto. Sin embargo el riesgo de recaída sigue constituyendo una verdadera amenaza que acompaña todo el proceso de desintoxicación-deshabituación-inserción o reinserción social. En efecto, el escollo de la recaída puede presentarse en cualquier momento del proceso terapéutico y, el reto que supone la recaída, se amplifica cuando los fracasos terapéuticos se acumulan a lo largo de la vida del paciente y más, todavía, allí donde la red social de contención y ayuda está debilitada o es inexistente.

La recaída precoz (12 primeras semanas) tras el abandono de la bebida puede relacionarse con los efectos reforzadores del alcohol ${ }^{18}$. Dichos efectos reforzadores, tanto los positivos tales como la euforia y la sensación de bienestar, así como los negativos tales como el alivio del malestar psicofísico y emocional, mitigar los síntomas de abstinencia o posibles trastornos psiquiátricos concomitantes, han sido ampliamente estudiados relacionando el consumo crónico de alcohol y los sistemas dopaminérgico, gabaérgico, glutamatérgico, serotoninérgico y opioidérgico. Se ha postulado tanto la relación entre recaída y craving, particularmente en las primeras etapas de la abstinencia ${ }^{19}$, como también la asociación entre reducción del craving y reducción en el consumo de alcohol ${ }^{20}$ y además la posibilidad de que fármacos eficaces en la reducción del craving lo puedan ser, también, en el tratamiento del alcoholismo ${ }^{21,22}$. Además un factor, entre otros, que aumentaría la vulnerabilidad para la recaída precoz sería la mayor severidad de la dependencia ${ }^{23}$.

La desintoxicación de alcohol es un procedimiento terapéutico que tiene por objetivo facilitar el cese del consumo alcohólico sin repercusiones negativas para el paciente; en realidad constituye la primera fase de la deshabituación alcohólica y básicamente podría definirse como la "prevención y tratamiento del síndrome de abstinencia alcohólica"16 en cualquiera de sus formas y grados. La desintoxicación se hace necesaria porque "el consumo crónico de alcohol produce fenómenos de neuroadaptación, fenómenos de sensibilización y efectos neuroendocrinos. Los fenómenos de neuroadaptación están relacionados con la tolerancia y la abstinencia del alcohol y se acompañan de una modificación de los efectos reforzadores del alcohol"24. En definitiva, en el anhelo (craving) de alcohol y en la vuelta compulsiva a su consumo que predice la recaída intervendría la compleja interacción de estos mecanismos neuroadaptativos, neuro-endocrinológicos y de sensibilización.

La desintoxicación puede llevarse a cabo en un entorno hospitalario o de manera ambulatoria; la desintoxicación ambulatoria reúne algunas ventajas tales como incentivar la responsabilidad del paciente y su implicación en el tratamiento, un coste económico menor y mantener la continuidad con las siguientes fases de la rehabilitación alcohólica, y también muestra algunos inconvenientes como la dificultad en controlar el cumplimiento farmacológico, una menor seguridad para el paciente y un elevado índice de fra$\operatorname{casos}^{25}$. Nosotros elegimos la modalidad de desintoxicación ambulatoria con tratamiento de observación directa parcial en esta muestra limitada de pacientes, a fin de comprobar de manera preliminar la eficacia de esta alternativa terapéutica.

Los tratamientos de observación directa (TOD), también llamados directamente supervisados (TDO) son un procedimiento terapéutico que consiste en la administración de fármacos bajo la supervisión de personal sanitario, durante un tiempo determinado y para el tratamiento de una determinada enfermedad, que se realiza con el fin de asegurar el cumplimiento terapéutico y aumentar la adherencia en orden a mejorar los resultados de la intervención y que ha ido evolucionando en complejidad a lo largo del tiempo; se adoptó por primera vez en los años setenta para el tratamiento de la tuberculosis y forma parte de una de las cinco estrategias incluidas en el programa DOTS (Directly Observed Treatment, Short Course) de la OMS 26, 27, 28, 29,30. Varios estudios y ensayos clínicos avalan ${ }^{31,32,33}$ y otros critican su eficiencia ${ }^{34}$. Sin embargo, por lo que se refiere a pacientes con dependencia de alcohol, con el propósito de favorecer el cumplimiento y la adherencia, no conocemos la existencia de estudios, ni siquiera preliminares, que hayan empleado esta modalidad de intervención terapéutica. Nuestro estudio limitado a 18 pacientes y doce semanas de seguimiento, incluye la observación directa de la toma de una dosis diaria de medicación, durante cinco días por semana como máximo, frecuencia de observación directa que experimenta una reducción paulatina, según se detalla en el apartado "Métodos", a lo largo de las doce semanas de seguimiento de los pacientes.

Por adherencia establecimos el cumplimiento de las pautas pactadas de tratamiento: acudir a la Unidad de Conductas Adictivas (UCA) con la periodicidad convenida y que se explicita en el apartado "Métodos", ingerir en ella una dosis de medicación, recibir las dosis restantes para el día o para el fin de semana y autoadministrárselas, comprometerse a mantener la abstinencia a alcohol y a cualquier otra droga, a excepción del tabaco si eran fumadores y no habían decidido abandonar su consumo y, finalmente, cumplir el acuerdo de manifestar la violación tanto de la 
abstinencia como de cualquiera de estos compromisos. Si el paciente omitía alguna dosis de medicación y permanecía abstinente se mantenía la consideración de "adherencia terapéutica", también se consideraba "adherencia" si el paciente reiniciaba el consumo de alcohol pero seguía acudiendo a consulta, en este caso, naturalmente, se revisaba la medicación y se ajustaban los objetivos terapéuticos. La "adherencia" se perdía cuando el paciente abandonaba, dejaba de acudir a la UCA los días señalados y convenidos, excepto por una razón de fuerza mayor justificada y en este caso por un periodo nunca superior a los dos días.

El objetivo de este trabajo es estudiar de forma preliminar si una Unidad de Conductas Adictivas (UCA) puede, ambulatoriamente, desintoxicar y disminuir el riesgo de recaída precoz (doce semanas) en pacientes alcohólicos, con una dependencia orgánica establecida, con un síndrome de abstinencia grave, con múltiples tratamientos previos y que no habían conseguido mantener la abstinencia ni adherencia a tratamiento durante un periodo continuado de tres meses en los últimos dos años, y hacerlo mediante un modelo de abordaje multimodal médico-psicológico y de enfermería basado en una visita diaria breve y la supervisión de la toma de una dosis del tratamiento farmacológico, que podía incluir aversivos o naltrexona, confiando el resto de dosis del día y toda la medicación del fin de semana a los pacientes para que se las autoadministraran.

\section{MÉTODOS}

La muestra de pacientes del estudio se compone de 18 individuos, elegidos al azar, de entre los que solicitaron tratamiento en una Unidad de Conductas Adictivas (UCA) siempre que cumplieran las siguientes características:

- Historia de alcoholismo igual o mayor de 5 años.

- Múltiples tratamientos previos (al menos cuatro) incluida alguna desintoxicación hospitalaria y/o ambulatoria.

- En los últimos 24 meses no les consta a los pacientes, ni a su red social si la tenían, haber podido mantener abstinencia continuada durante 12 semanas o más.

- Síndrome de dependencia de alcohol siguiendo los criterios establecidos por la CIE-10.

- En el MALT-O (Münchner Alkoholismus Test-Objetivo) obtener una puntuación indicativa de dependencia orgánica del alcohol.

- Presentar síndrome de abstinencia grave medido con CIWA-Ar (Clinical Institute Withdrawal Assessment of Alcohol) en su versión española.
- Red social deficiente, incontinente o inexistente.

- Se desestimaban para inclusión en este estudio preliminar los pacientes con antecedentes o sospecha clínica de patología psiquiátrica de la esfera psicótica, trastornos de la personalidad y los trastornos del espectro afectivo bipolar.

A continuación (Tabla 1) se expresa la edad media y la distribución por sexos de la muestra.

\section{Tabla 1. Distribución por edad media y sexos.}

\begin{tabular}{|c|c|}
\hline Edad media de la muestra & Distribución por sexos \\
\hline 42.5 años (29-61 años) & $\begin{array}{c}\text { Mujeres: } 3 \text { (18\%); Hombres } 15 \\
(82 \%)\end{array}$ \\
\hline
\end{tabular}

Las puntuaciones medias obtenidas en el MALT-O fueron: 16.46 (12-20), una puntuación igual o superior a 11 equivale a alcoholismo

Las puntuaciones medias obtenidas en el CIWA-Ar fueron: 27.05 (21-36), por encima de 20 se considera síndrome de abstinencia grave.

El circuito asistencial se realizó del siguiente modo:

1. Después de la evaluación inicial que incluía la exploración física básica, analítica de sangre y orina con detección de tóxicos y pruebas serológicas para hepatitis y $\mathrm{VIH}$, pruebas de la función tiroidea y entrevista clínica sobre antecedentes y tratamiento de psicosis, trastorno bipolar y trastornos de la personalidad, a todos los pacientes se les practicó una desintoxicación ambulatoria que sistemáticamente se iniciaba un lunes e incluía una visita diaria (de lunes a viernes) a la Unidad de Conductas Adictivas (UCA). En dicha visita se valoraba la situación clínica global de los pacientes, la tolerancia y cumplimiento medicamentoso y se interrogaba sobre la abstinencia, además de ofrecerles apoyo psicológico; todo ello en un formato breve de una duración aproximada de 8-10 minutos por paciente y día. La medicación se dividió en tres dosis diarias y en la visita, mediante supervisión y observación directa, ingerían una dosis, entregándoles las dos dosis restantes. Al llegar el viernes, quinto día de la desintoxicación ambulatoria, se entregaban al paciente seis unidosis extra, tres para el sábado y tres para el domingo. Durante la primera semana los pacientes permanecían en reposo relativo domiciliario. Esta situación, según los casos, se alargó hasta los 15 días. Las dosis de medicación entregadas a los pacientes no estaban sujetas a supervisión. 
2. Posteriormente seguían acudiendo cada día (de lunes a viernes) a la UCA procediendo del mismo modo hasta completar cuatro semanas de tratamiento; aunque, a partir de los primeros 15 días, los pacientes que tuvieron posibilidad de realizar alguna actividad laboral (trabajos temporales en la agricultura, o contratas de protección social en algún Ayuntamiento), podían elegir presentarse en la UCA a primera o a última hora de la mañana para no interferir con su jornada laboral.

3. Las semanas quinta y sexta, los pacientes recibían tratamiento de supervisión y observación directa tres días por semana, y se les seguían preparando unidosis para los días en que no debían presentarse en la UCA.

4. Las semanas séptima y octava acudían sólo dos veces por semana a la UCA.

5. De la semana novena a la doceava iban a la UCA una vez por semana.

6. El tratamiento farmacológico podía incluir: benzodiazepinas, antidipsotrópicos, naltrexona, anticomiciales, antipsicóticos y antidepresivos, según las necesidades individualmente consideradas de cada paciente.

7. La pauta farmacológica estándar de desintoxicación estaba compuesta por: lorazepam en dosis variables ajustadas a diario, si era preciso, según la sintomatología abstinencial medida sobre la basal obtenida del CIWA-Ar y vitaminoterapia. La utilización, además, de otros fármacos seguía el siguiente criterio: los anticomiciales y antipsicóticos dependían de la clínica (ansiedad y / o insomnio, y / o CIWA-Ar superior a 15, y / o abandono de la benzodiazepina utilizada en la desintoxicación o consumo previo de benzodiazepinas), o de la respuesta previa en otras desintoxicaciones; los antidepresivos no se añadían antes de trascurridas 4-5 semanas de inicio del tratamiento, y únicamente si la clínica era compatible con un trastorno depresivo o ansioso susceptible de tratamiento con ISRS; la naltrexona y los aversivos se recomendaban según el nivel de craving.

8. La medida de craving (ansia o anhelo de consumo) se obtuvo con una Escala Analógica Visual (EVA), ciega para el paciente y numerada para el explorador. La estimación del craving se solicitaba dos veces por semana durante la desintoxicación (15 primeros días) y únicamente se consideraba si superaba el $50 \%$, es decir 5 puntos sobre 10 en la EVA, entonces se ajustaba al alza el tratamiento farmacológico, y cuando esa barrera del $50 \%$ se rebasaba en dos mediciones consecutivas se sugería la utilización de antidipsotrópicos o naltrexona, opción que se dejaba abierta a la preferencia de los pacientes. Durante las 10 semanas restantes se media el craving, mediante la EVA, una vez por semana.

9. El apoyo psicológico brindado durante todo el proceso consistió en intervenciones motivadoras, en crisis y orientadas al mantenimiento de la adherencia a tratamiento, todo ello con un formato breve y que se llevaba a cabo por todos los miembros del equipo (facultativos, psicólogos y enfermería); las situaciones de crisis, cuando se detectaban, se abordaban mediante una intervención psicológica específica de orientación cognitivo-conductual.

10. El criterio de recaída que establecimos fue el reinicio del consumo de alcohol aún en pequeñas cantidades y aunque este no fuese compulsivo. El motivo para elegir este criterio venía dado por la historia adictiva de los pacientes, la cual aconsejaba mantener la abstinencia debido al alto riesgo de pérdida de control. En cada visita se interrogaba a cada paciente sobre el consumo de alcohol y el cumplimiento medicamentoso. El paciente había sido informado, y se le reiteraba constantemente, de que era indispensable que nos informara abiertamente de cualquier consumo de alcohol, lo cual no le supondría ninguna penalización ni abandono de tratamiento ni de las atenciones que estaba recibiendo. Dado que no existe ningún criterio objetivo capaz de avalar con carácter de absoluta certeza la abstinencia en medio ambulatorio, decidimos apostar por la complicidad y sinceridad del paciente desde la proximidad en la relación terapéutica.

11. Fueron descartados de la muestra los pacientes con abuso o adicción a otras sustancias excepto benzodiazepinas y tabaco y también aquellos con ludopatía activa; los candidatos con historia de trastornos del espectro psicótico y bipolar, los pacientes con intentos previos de suicidio así como trastornos de personalidad también fueron excluidos de este estudio preliminar; así mismo cuando los pacientes carecían de dichos antecedentes pero en la entrevista clínica previa al inicio del tratamiento se sospechaban estas patologías y riesgos con fundamento, se desestimaron para este estudio piloto. No se realizó una exploración y seguimiento instrumental objetivo de la patología psiquiátrica de la esfera ansiosa y depresiva. Pero dada la importancia y prevalencia de esta patología psiquiátrica en el alcoholismo y el hecho de que esta comorbilidad puede contribuir a aumentar el riesgo de recaída ${ }^{21}$, se practicó un seguimiento clínico en todas las visitas, tal y como queda indicado en el punto 7 de este apartado "Métodos".

12. A los pacientes reclutados para este estudio se les practicaba, de manera rutinaria, una analítica de orina semanal y sorpresiva para drogas de abuso, si se obtenían dos positivos en cualquier momento del seguimiento eran excluidos del estudio. 


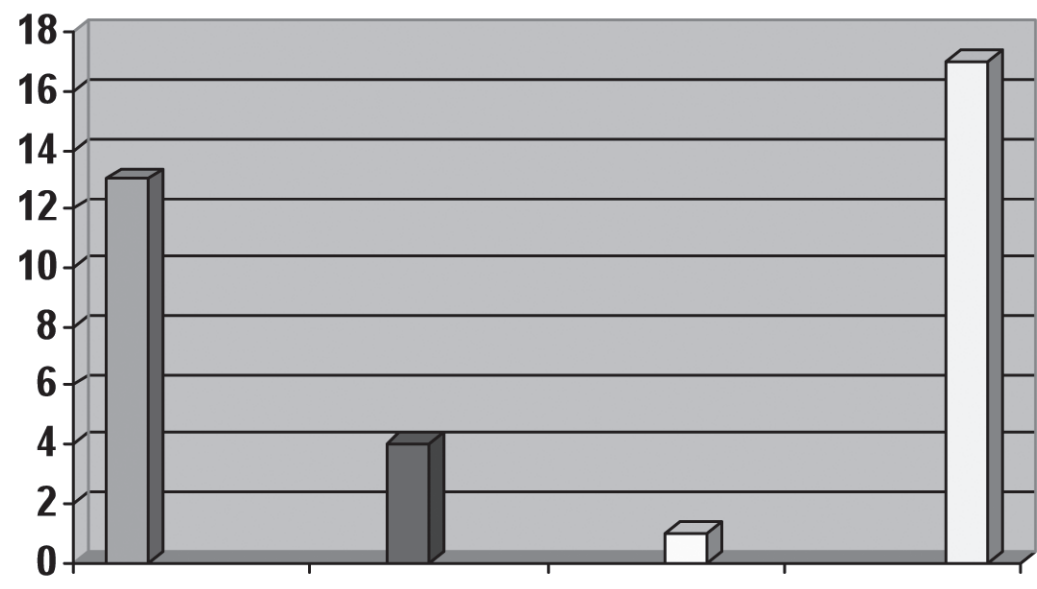

\section{$\square$ abstinencia $\square$ recaída $\square$ abandono $\square$ adherencia}

Figura 1. Resultados a las 12 semanas (N: 18).

\section{RESULTADOS}

De los dieciocho pacientes que fueron incluidos en este estudio, a las doce semanas, trece (72\%) permanecían abstinentes, cuatro (22\%) recayeron, uno abandonó y diecisiete (94\%) se mantenían adheridos a tratamiento (Figura 1).

Las características de los 13 pacientes abstinentes (Tabla 2), de los 4 que recayeron y del que abandonó (Tabla 3), eran semejantes.

Tabla 2. Pacientes abstinentes a las 12 semanas.

\begin{tabular}{|c|c|}
\hline Abstinentes a las $\mathbf{1 2}$ semanas & $\mathbf{N}=\mathbf{1 3}(\mathbf{7 2 , 2} \mathbf{\% )}$ \\
\hline Mujeres & $2(16 \%)$ \\
\hline Hombres & $11(84 \%)$ \\
\hline Edad media & 43.5 años \\
\hline MALT-O medio & 16.92 \\
\hline CIWA-Ar medio & 28.07 \\
\hline
\end{tabular}

Tabla 3. Recaídas y abandono a las 12 semanas.

\begin{tabular}{|c|c|}
\hline $\begin{array}{c}\text { Recaídas y abandono a } \\
\text { las } \mathbf{1 2} \text { semanas }\end{array}$ & $\mathbf{N}=\mathbf{5}(\mathbf{2 7 , 7} \%)$ \\
\hline Mujeres & $1(20 \%)$ \\
\hline Hombres & $4(80 \%)$ \\
\hline Edad media & 41.6 años \\
\hline MALT-O medio & 16 \\
\hline CIWA-Ar medio & 25.4 \\
\hline
\end{tabular}

A pesar de la gravedad del síndrome de abstinencia detectado por el CIWA-Ar no hubo complicaciones que obligaran al ingreso hospitalario de ningún paciente; todos ellos pudieron completar la desintoxicación ambulatoria de manera satisfactoria con excepción del caso que abandonó, y que lo hizo durante la primera semana de la desintoxicación.

El $88 \%$ de los pacientes presentaron un craving superior al $50 \%$ en dos estimaciones consecutivas durante la desintoxicación (primeros 15 días), y fue en este periodo donde se inició en tratamiento con aversivos y naltrexona, el resto $22 \%$ no superaron el craving establecido del $50 \%$ en dos ocasiones consecutivas. El resto del periodo de tratamiento, 10 semanas, la EVA para medir el craving, no superó en ningún paciente el límite establecido del $50 \%$ en dos ocasiones consecutivas (tabla 4).

Tabla 4. Craving ( $\mathbf{N}=18$ ).

\begin{tabular}{|c|c|c|}
\hline $\begin{array}{c}\text { Periodo de desintoxicación } \\
\text { (hasta 15 días) }\end{array}$ & $\begin{array}{c}>50 \% \\
(88 \%)\end{array}$ & $\begin{array}{c}<50 \% \\
(22 \%)\end{array}$ \\
\hline 10 semanas restantes & \multicolumn{2}{|c|}{$<50 \%$} \\
\hline
\end{tabular}

La pauta farmacológica estándar para la desintoxicación fue la siguiente: lorazepam (las dosis oscilaron entre 7 y 9 mg/día) que se administró en pauta descendente durante el periodo de desintoxicación que duró 15 días, y vitaminoterapia (B1 y B12 más ácido fólico). En el $88 \%$ de la muestra (16 pacientes), durante los 15 días del periodo de desintoxicación ambulatoria, la puntuación en la EVA para la estimación del craving superaba el $50 \%$ (5/10) en dos ocasiones consecutivas, entonces se ofreció a los pacientes la posibilidad de fármacos aversivos (disulfiram) o naltrexona. En el $77 \%$ (14 pacientes) se utilizó disulfiram oral y en 2 pacientes $(11 \%)$ se usó naltrexona. 
Los anticomiciales fueron necesarios en 12 pacientes, el 66,6 \% de la muestra: topiramato: (se administró a 6 pacientes, dosis media $150 \mathrm{mg}$ ), oxcarbazepina: (se recetó a 6 pacientes, dosis media $900 \mathrm{mg}$ ); los antipsicóticos se utilizaron en 9 pacientes, el $50 \%$ de la muestra: risperidona (se prescribió en 5 pacientes, dosis media $2 \mathrm{mg}$ ), olanzapina (la recibieron 4 pacientes, dosis media $10 \mathrm{mg}$ ); los ISRS (escitalopram: dosis media $15 \mathrm{mg}$ ) se prescribieron a 10 pacientes.

El grado de adherencia conseguida, (94\%) en los pacientes de nuestra muestra, favoreció que aquellos más deteriorados y con menores recursos y posibilidades pudieren beneficiarse, no solo de algunos programas sociales sino también de los servicios básicos de salud, con los consiguientes beneficios para su calidad de vida. El paciente que abandonó durante la primera semana lo hizo después de sugerirle antidipsotrópicos o naltrexona cuando su craving, medido mediante EVA, superaba el 50 \% en dos mediciones consecutivas; primero manifestó aceptar antiodipsotrópicos pero la realidad fue que dejó de acudir a consulta.

\section{DISCUSIÓN}

Creemos que la modalidad de TOD utilizada por nosotros resulto beneficiosa para 13 de los 18 pacientes de la muestra que consiguieron, sin complicaciones, la desintoxicación ambulatoria y mantuvieron la abstinencia durante 12 semanas, y favoreció también que 17 de 18 pacientes del estudio permanecieran adheridos a tratamiento durante el mismo periodo de tiempo.

En este trabajo y para el diagnóstico de alcoholismo de los pacientes, obviamos el MALT-S. Tal decisión se basó en el hecho de que en el MALT-O, los 18 pacientes de la muestra, obtuvieron puntuaciones superiores a los 11 puntos, punto de corte para el diagnóstico de alcoholismo. Por ello entendimos que habiendo obtenido en los 7 ítems de que consta el MALT-O una puntuación superior al punto de corte estimado para diagnosticar alcoholismo, junto con la historia clínica de los pacientes en relación con el alcohol, quedaba suficientemente acreditado no sólo el diagnóstico sino también la dependencia psicológica del alcohol.

Por lo que se refiere a los tratamientos farmacológicos para la desintoxicación elegimos el lorazepam, pautado en tres tomas diarias y ajustado según CIWA-Ar igual que recomiendan Guardia Serecigni et al. ${ }^{55}$; las dosis fueron decreciendo hasta su retirada a los 15 días. Elegimos lorazepam, porque es una benzodiazepina manejable ambulatoriamente por vía oral cuya farmacocinética describe una vida media de
10-20 horas y posee un metabolismo por glucuronidación hepática ${ }^{35}$. Todos nuestros pacientes recibieron vitaminoterapia como tratamiento complementario del síndrome de abstinencia alcohólica tal y como aconsejan Thomson y Marshall ${ }^{36}$. Nosotros empleamos suplementos de vitamina B1, B12 y ácido fólico: para prevenir la encefalopatía de Wernicke usamos dosis de 300 mg de tiamina (vitamina B1) por vía oral cada 12 horas los siete primeros días, pasando a partir del octavo día a 300 mg cada día, hasta completar 21 días de tratamiento; para cubrir las posibles carencias de cianocobalamina (vitamina B12) y sobre todo de ácido fólico que a menudo acompañan al consumo crónico de alcohol, teniendo en cuenta que las necesidades mínimas diarias son aproximadamente de $2.5 \mathrm{mcg}$ de cianocobalamina y de 50 mcg de ácido fólico, incluimos por vía oral, 6 mcg de cianocobalamina junto a 1,2 mg de ácido fólico ${ }^{37}$, fraccionados en tres dosis y durante 45 días.

Hughes y Cook $^{38}$ realizaron una revisión sobre 34 estudios realizados sobre aversivos del alcohol entre los años 1967-1995, y encontraron no solo problemas metodológicos sino también fallos estadísticos en la realización de estos estudios, sin embargo parece aceptarse que la eficacia de los fármacos aversivos mejora y su utilización reduce el número de días en los que el paciente ingiere alcohol ${ }^{39}$ si se consigue aumentar la adhesión al tratamiento por supervisión de un familiar o persona responsable ${ }^{4}$, o asociando el disulfiram a otros programas de tratamiento tanto psicosocial ${ }^{40}$ como farmacológico ${ }^{41}$. Nosotros incluimos el disulfiram oral en el tratamiento de observación directa cuando por causa del craving, tal y como se explica en Métodos, se ofrecía a los pacientes disulfiram o naltrexona y en nuestra muestra el 77 \% (14 pacientes) prefirieron la administración de disulfiram. $Y$ actuamos de este modo porque, aunque no existe ningún estudio que haya evaluado el posible efecto anticraving del disulfiram, la experiencia subjetiva de algunos pacientes que toman disulfiram de manera continuada es que no experimentan deseos de beber ni tampoco idas rumiatorias entorno a la posibilidad de beber ${ }^{55}$.

La naltrexona actuaría como fármaco anticraving influyendo sobre el sistema opioide endógeno y disminuyendo la capacidad reforzante del alcohol y en consecuencia el deseo de consumo ${ }^{42}$, y modulando la actividad del sistema dopaminérgico y su relación con el craving ${ }^{43}$. Sobre la naltrexona existen datos contradictorios, de entre los cuales, citaremos tan sólo tres estudios recientes a modo de ejemplo, puesto que no es el propósito de este trabajo hacer una revisión exhaustiva sobre este asunto. Por un lado ni el porcentaje de días de consumo, ni el número de bebidas alcohólicas ingeridas por días de consumo sería significativamente diferente frente al placebo ${ }^{44}$; por otro disminuiría el número de recaídas en pacientes 
que prueban el alcohol, el número de días de consumo y las cantidades totales de alcohol ingerido ${ }^{45}$, así como conseguiría una mayor reducción del craving. Otro estudio compara disulfiram y naltrexona y concluye que disulfiram lograría disminuir más el número de recaídas, el de consumiciones por día de consumo y aumentaría el número de días en que los pacientes se mantendrían abstinentes ${ }^{46}$. En nuestra muestra el $11 \%$ (2 pacientes) prefirieron naltrexona a disulfiram para tratar de reducir el craving de alcohol.

Los otros fármacos utilizados por nosotros: anticomiciales (topiramato y oxcarbazepina), antipsicóticos (risperidona y olanzapina) e ISRS (escitalopram), respondieron a las necesidades expresadas en el punto de Métodos. Respecto de los antiepilépticos y el tratamiento de las adicciones, Pereiro et $\mathrm{al}^{47}$. evalúan el número de recetas de antiepilépticos extendidas y comprueban que su número se "ha duplicado entre los años 2002 y 2004, manteniendo una clara tendencia al incremento también en el 2005, que se espera que continúe". Ello abunda en el interés creciente de estos fármacos y en el papel que pueden tener en el control del craving, el consumo de sustancias y la impulsividad. En este mismo sentido varios estudios $48,49,50,51,52,53,54,55,56$ analizan el papel de estos fármacos y su utilidad en la desintoxicación, en el control de la impulsividad, el craving y algunos trastornos psiquiátricos asociados, así como sobre la reducción en el consumo de alcohol y otras sustancias adictivas. Nosotros usamos topiramato en 6 pacientes y oxcarbazepina en otros 6 pacientes, también, para evitar el uso de benzodiazepinas más allá del periodo de desintoxicación. EI ISRS (escitalopram) lo prescribimos a 10 pacientes y lo hicimos cuando, transcurridas 4-5 semanas del inicio del tratamiento, persistía una clínica (CIE-10 o DSM-IV-TR) compatible con un trastorno ansioso o depresivo. Elegimos emplear un ISRS porque han demostrado sobradamente su papel en los trastornos depresivos y, además, constituyen en la actualidad el tratamiento de primera línea de muchos trastornos de ansiedad, a la vez que ofrecen cierta protección para evitar la recaída ${ }^{57}$ en dichos trastornos, lo cual posee un valor inestimable sobre todo para los pacientes alcohólicos porque presentan, con cierta frecuencia, un consumo abusivo de benzodiazepinas ${ }^{58}$. Varios estudios han permitido conocer diferentes aplicaciones de los antipsicóticos risperidona y olanzapina además de su indicación para los trastornos psicóticos. Así, la risperidona a dosis bajas, podría representar una alternativa terapéutica útil en el manejo del trastorno por ansiedad generalizado ${ }^{59}$; y la olanzapina se ha mostrado beneficiosa en el trastorno de estrés post-traumático y el trastorno límite de personalidad entre otros ${ }^{60}$. Nosotros usamos estos fármacos en 9 pacientes ( 5 risperidona y 4 olanzapina), a dosis bajas (la dosis media de risperidona fue de $2 \mathrm{mg}$ y la de olanzapina $10 \mathrm{mg}$ ), especialmente para tratar la ansiedad y el insomnio y evitar el uso de benzodiazepinas más allá del periodo de desintoxicación.

En nuestro estudio, mediante el CIWA-AR que permite medir de forma útil la presencia y la gravedad del síndrome de abstinencia alcohólica ${ }^{55}$, hallamos en nuestra muestra una puntuación media de 27.05 (20 es el punto de corte a partir del cual se considera síndrome de abstinencia grave), factor éste que privilegiaría la elección de la desintoxicación hospitalaria frente a la ambulatoria25, 55; a pesar de todo lo cual, hay que destacar que no se produjo en ninguno de los 17 pacientes, que completaron la desintoxicación ambulatoria, ningún acontecimiento adverso que requiriera su ingreso hospitalario.

No existen estudios sobre los tratamientos de observación directa en la desintoxicación y prevención temprana de recaídas (tres meses) de pacientes diagnosticados de trastorno por dependencia del alcohol. Los resultados de este estudio preliminar indican que se consiguió un buen porcentaje de adherencia terapéutica $94 \%$, con tan solo un abandono, que la modalidad de TOD que empleamos, en el contexto del tratamiento multimodal médico-psicológico y de enfermería, resultó un procedimiento terapéutico seguro y se obtuvo una tasa de abstinencia declarada a las 12 semanas del $72 \%$. Sin embargo deben tenerse en cuenta sus limitaciones: no se trata de un ensayo controlado, se realiza con una muestra reducida compuesta por 18 pacientes, contiene un seguimiento limitado (12 semanas), se excluye a los pacientes con patología psiquiátrica, no se realiza un seguimiento instrumental de la patología ansiosa y depresiva y se considera recaída el consumo alcohol declarado por el paciente, aunque fuera en pequeñas cantidades. A pesar de todo, el trabajo que presentamos es una primera aproximación a la posible utilidad de los TOD para el tratamiento del trastorno por dependencia del alcohol y la prevención temprana de recaídas. Sus resultados han de mirarse con prudencia, y esperar que ensayos controlados permitan confirmar su eficiencia en la desintoxicación ambulatoria de pacientes alcohólicos. Nuestra intención ha sido contribuir a evitar lo que tan acertadamente describe Cañuelo61 para muchos pacientes que, inveteradamente "impermeables a los estímulos de la posible abstinencia", pueden mostrarse "escépticos y apáticos ante las ofertas de una sociedad excluyente a su situación", a la que muchas veces responsabilizan y de la que "recelan, prefiriendo seguir siendo como son y estar donde están" antes que aspirar a lo "que podría proporcionarles otro fracaso que, seguramente, no saben si podrían encajar después de todos los acumulados". 


\section{REFERENCIAS}

1. Miller NS, Gold MS. Alcohol. Barcelona: Ediciones en Neurociencias, Citran, Fisp; 1993 [original 1991].

2. Gual Solé A, Monras Arnau M. Factores que influyen en la cumplimentación del tratamiento del síndrome de dependencia alcohólica. En: García-Rodriguez JA, López Sánchez C, coordinadores. Manual de Estudios sobre el alcohol. Madrid: Edaf, S.A; 2001. p. 203-38.

3. Sociedad Española de Psiquiatría. Consenso de la Sociedad Española de Psiquiatría sobre diagnóstico y tratamiento del alcoholismo y otras adicciones. Barcelona: Euromedice; 2003.

4. Balcells M, Gual A. Farmacoterapia de la deshabituación alcohólica. Nuevos fármacos, nuevos conceptos. Adicciones 2002; 14 Supl 1: 439-48.

5. Guardia J, Bobes J, Becoña E, Fernández Miranda JJ, Furió JL, Pereiro C, et al. El impacto del alcohol en España y Europa. Adicciones 2007; 69-70.

6. Organización Mundial de la Salud. Décima revisión de la Clasificación Internacional de las Enfermedades. Trastornos mentales y del comportamiento. Madrid: Meditor; 1992.

7. Feuerlein W, Ronger C, Küfner KA. Diagnose des Alkololismus: Der Münchner Alkolismustes (MALT). Münch Med Wochf 1977; 119: 1275-82.

8. Rodríguez-Martos A, Suárez R. MALT (Münchner Alkoholismus Test). Validación de la versión española de este test para el diagnóstico del alcoholismo. Rev Psiquiatr Psicol Med 1984; 16: 421-32.

9. Sáiz PA, G-Portilla $M^{a} P$, Paredes $M^{a} B$, Bascarán $M^{a} T$, Bobes J. Instrumentos de evaluación en alcoholismo. Adicciones 2002; 14 Supl 1: 387-403.

10. Ewing JA, Rouse BA. Identifying the hidden alcoholic. Presented at the Twenty-Ninth Internacional Congress on Alcohol and Drug Dependence. Sydney, Australia, February 1970.

11. Mayfield D, McLeod G, Hall P. The CAGE questionnaire validation of a new alcoholism screening instrument. Am J Psych 1974; 131: 1121-22.

12. Rodríguez Martos A, Navarro R, Vecino C, Pérez R. Validación de los cuestionarios KFA (CBA) y CAGE para el diagnóstico del alcoholismo. Drogalcohol 1986; 11 : 132-39.

13. Freuerlein W. Kurzfragenbogen für Alkoholdefährdete (KFA): eine empirische Analyse. Arch Psychiatr Nervenkr 1976; 222: 139-152.

14. Williams D, Lewis J, McBride A. A comparasion of rating scales for the alcohol withdrawal syndrome. Alcohol and Alcoholism 2001; 36: 104-8.

15. Sullivan JT, Sykora K, Schneiderman J, Naranjo CA, Sellers EM. Assessment of alcohol withdrawal: the revised Clinical Institute Withdrawal Assessment for Alcohol Scale (CIWA-Ar). Br J Add 1989; 84: 1353-57.

16. Soler PA, Guasch M. Tratamiento Farmacológico: la desintoxicación. Adicciones 2002; 14 Supl 1:421-37.

17. Trujols J, Tejero A, Casas M. El fenómeno de las recaídas en el tratamiento de las conductas adictivas y su repercusión en la dinámica asistencial. Adicciones 1996; 8:349-67.

18. Self DW, Nesttler EJ. Relapse to drug-seeking: neural and molecular mechanisms. Drug and Alcohol Dependence 1998; 51: 49-60.

19. Anton RF, Moak D, Latham PK: The Obsessive Compulsive Drinking Scale: Assessing outcome in alcoholism treatment studies. Arch. Gen. Psychiatry 1996; 53: 225-31.

20. Monti PM, Rohsenow DJ, Rubonis A, Niaura RS, Sirota A, Colby $S$, et al. Cue exposure with coping skills treatment for male alcoholics. A preliminary investigation. Journal of Consulting and Clinical Psychology 1993; 61: 1011-19.

21. Swift RM. Drug therapy: Drug therapy for alcohol dependence. The New England Journal of Medicine. 1999a; 340: 1482-90.

22. Swift RM. Medications and alcohol craving. Alcohol Research \& Health 1999b; 23: 207-13.

23. Guardia J, Catafau AM, Batlle F, Martian JC, Segura L, Gonzalvo B, et al. Striatal dopaminergic D2 receptor density measured by (123I) lodobenzamide SPECT in the prediction of treatment outcome of alcoholdependent patients. Am. J. Psychiatry 2000; 157: 127-29.

24. Guardia Serecigni J. Deshabituación del alcoholismo y prevención de recaídas. En: Casas Brugué $M$, Collazos Sánchez F, Ramos-Quiroga JA, Roncero Alonso C. Psicofarmacología de las drogodependencias. Bercelona: Esteve; 2002. p. 65-91.

25. Valderrama Zurián JC, La desintoxicación de la dependencia alcohólica. En: Casas Brugué M, Collazos Sánchez F, Ramos-Quiroga JA, Roncero Alonso C. Psicofarmacología de las drogodependencias. Bercelona: Esteve; 2002. p. 39-61.

26. Grupo de estudio del taller de 1999 de la Unidad de Investigación en tuberculosis de Barcelona. Documento de consenso sobre tratamientos directamente observados en tuberculosis. Med Clin 2000; 115: 749-57.

27. Macq JMC, Theobald S, Dick J, Demeble M. An exploration of the concept of directly observed treatment (DOT) for tuberculosis patients: from a uniform to a customised approach. Int J Tuberc Lung Dis 2003; 7:103-9.

28. Ministry of Health. 2002. Guidelines for tuberculosis Control in New Zealand 2003. Chapter 4: Adherence to treatment. Wellington: Ministry of Health; 2002. p. 1-24.

29. Bayer R, Wilkinson D. Directly observed terapy for tuberculosis: history an idea. Lancet 1995; 345: 1545-8.

30. World Health Organization. An expanded DOTS framework for effective tuberculosis control. WHO Stop TB Department, Geneva, 2002 (WHO/CDS/ TB/2002.297).

31. Chaulk CP, Kazandjian VA. Directly observed therapy for treatment completion of pulmonary tuberculosis: Consensus statement of the public health tuberculosis guidelines panel. JAMA 1998; 279: 943-8. 
32. Borgdorf MW, Floyd K, Broekmans JF. Interventions to reduce tuberculosis mortality and transmission in low-and middle-income countries. WHO Bull 2002; 80: 217-27.

33. Baltussen R, Floyd K, Dye C. Cost effectiveness analysis of strategies for tuberculosis control in developing countries. BMJ 2005; 331: 1364-71.

34. Volmink J, Garner P. Directly observed therapy for treating tuberculosis. The Cochrane Database of Systematic Reviews 2006, Issue 2. Art. No.: CD003343. DOI: 10. 1002/14651858.CD003343.pub2.

35. Ashok R, Sheehan D. Benzodiazepinas. En: Schatzberg AF, Nemeroff CB. Tratado de psicofarmacología. Barcelona: Masson; 2006 [original 2004]. p. 405-26.

36. Thomson AD, Marshall EJ. The treatment of patients at risk of developing Wernicke's encephalopathy in the community. Alcohol Alcohol. 2006 Mar-Apr; 41 (2): 159-67.

37. Babior BM, Bunn HF. Anemias megaloblásticas. En: Braunwald E, Fauci AS, Kasper DL, Hauser SL, Longo $D L$, Jameson JL, editores. Harrison, Principios de Medicina Interna. Madrid: McGraw-Hill-Interamericana de España; 2001 [original 2001]. 798-805.

38. Hughes JC, Cook CH. The efficacy of disulfiram; a review of outcome studies. Addiction 1997; 92 Supl 4: 381-395

39. Williams SH. Medications for treating alcohol dependence. American Family Physician. 2005.

40. Azrin NH, Sisson RW, Meyers R, Godley M. Alcoholism treatment by disulfiram and community reinforcement therapy. J Behav Ther Exp Psych 1982, 13: 105-12.

41. Landabaso MA, Iraurgi I, Sanz J, Fernández de Corres $B$, Ruiz de Apodaka J, Jiménez-Lerma JM, et al. Naltrexona más aversivo en alcohólicos refractarios a tratamiento. Psiquiatría Biológica 1997; 4: 5-8.

42. Antón RF, Kranzler HR, Meyer RE. Neurobehavioral aspects of pharmacotherapy of alcohol dependence. Clin Neuroscience 1995; 3: 145-154.

43. Samson HH, Harris RA. The neurobiology of alcohol abuse. TINS 1992; 13: 206-211.

44. Kristal JH, Cramer JA, Krol WF, Kira GF, Rosenheck RA. Naltrexone in the treatment of alcohol dependence. New Engl J Med 2001; 345: 1734-39.

45. Guardia J, Caso C, Arias F, Gual A, Sanahuja J, Ramirez $M$, et al. A double-blind, placebo-controlled study of naltrexone in the treatment of alcohol-dependence disorder. Results from a multicenter clinical trial. Alcohol Clin Exp Res 2002; 26 (9): 1381-1387.

46. De Sousa A, De Sousa A. A one-year pragmatic trial of naltrexone vs disulfiram in the treatment of alcohol dependence. Alcohol \& Alcoholism 2004; 39: 528-531.

47. Pereiro Gómes C, Carrera Machado I, Mejuto Val. Prescripción de antiepilépticos en el tratamiento de las adicciones en Galicia 2002-2006. Ponencia presentada a las XXXIV Jornadas Nacionales de Socidrogalcohol. Valencia, 22-24 de marzo de 2007.

45. Gimeno C, Castillo A, Llopis J, Pascual P. Oxcarbamazepina en la dependencia a la cocaína y alcohol: experiencia clínica. Ponencia presentada a las XXXIV Jornadas Nacionales de Socidrogalcohol. Valencia, 22-24 de marzo de 2007.

49. De la Fuente Darder B, Aparicio Aparicio A, De la Guía Castro Granados M, Lizaur Barrudo M, Mánchado López L. Eficacia de la Oxcarbamazepian en la deshabituación de adictos a cocaína y alcohol.Comunicación presentada a las XXXIV Jornadas Nacionales de Socidrogalcohol. Valencia, 22-24 de marzo de 2007.

50. Morillo-Velarde C, Cáceres D, Cabrera MV. Eficacia terapéutica de oxcarbamazepina sobre la impulsividad y el craving. Comunicación presentada a las XXXIV Jornadas Nacionales de Socidrogalcohol. Valencia, 22-24 de marzo de 2007.

51. Fernández Miranda JJ. Anticonvulsivantes en el tratamiento de la dependencia del alcohol: topiramato. Ponencia presentada a las XXXIV Jornadas Nacionales de Socidrogalcohol. Valencia, 22-24 de marzo de 2007.

52. Dorado ML, Llorens N, Palau C, Lópes I, Sánchez A, Malea A, et al. Eficacia del topiramato en la deshabituación de alcohol. Comunicación presentada a las XXXIV Jornadas Nacionales de Socidrogalcohol. Valencia, 22-24 de marzo de 2007.

53. Pino Serrano CJ. Estudio con 32 pacientes tratados con topiramato y su evolución a 12 meses. Comunicación presentada a las XXXIV Jornadas Nacionales de Socidrogalcohol. Valencia, 22-24 de marzo de 2007.

54. Jiménez-Arriero MA, Rodríguez-Jiménez R, Bagney $A$, Ponce G. Utilidad de los anticonvulsivantes en el tratamiento del alcoholismo. Ponencia presentada a las XXXIV Jornadas Nacionales de Socidrogalcohol. Valencia, 22-24 de marzo de 2007.

55. Guardia Serecigni J, Jiménez-Arriero MA, Pascual Pastor P, Fórez Menéndez G, Contell Guillamont M. Guía clínica para el tratamiento del alcoholismo. Socidrogalcohol 2007; 71.

56. Ait-Daoud N, Malcom RJ Jr, Johnson BA. An overview of medications for the treatment of alcohol withdrawal and alcohol-dependence with an emphasis on the use of older and newer anticonvulsants. Addict Behav. 2006 Feb 9.

57. Davidson JRT, Connor KM. Tratamiento de los trastornos de ansiedad. En: Schatzberg AF, Nemeroff CB. Tratado de psicofarmacología. Barcelona: Masson; 2006 [original 2004]. p. 1009-33.

58. Cornish JW, McNicholas LF, O'Brien CP. Tratamiento de los trastornos relacionados con sustancias. En: Schatzberg AF, Nemeroff CB. Tratado de psicofarmacología. Barcelona: Masson; 2006 [original 2004]. p. 1119-42.

59. Brawman-Mintzer O, Knapp RG, Nietert PJ. Adjuntive risperidone in generalizad anxiety disorder: A doubleblind, placebo-controlledudy. J Clin Psychiatry. 2005 Oct; 66 (10): 1321-5.

60. Schulz SC, Olson S, Kotlyar M. Olanzapina. En: Schatzberg AF, Nemeroff CB. Tratado de psicofarmacología. Barcelona: Masson; 2006 [original 2004]. p. 499-516.

61. Cañuelo B. Alcohol y exclusión social. Adicciones 2002; 14 Supl 1: 251-60. 